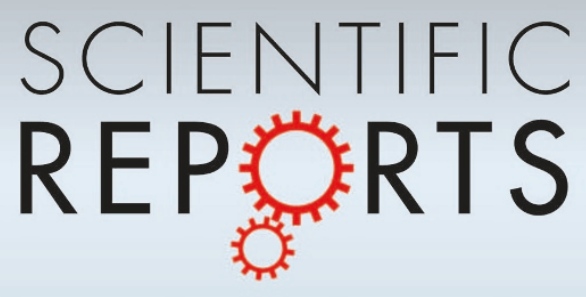

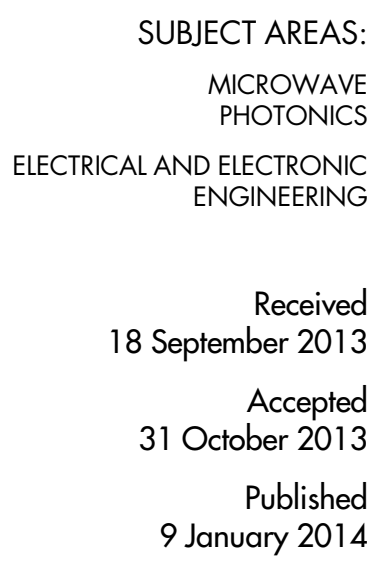

Correspondence and requests for materials should be addressed to W.L. (willoh@mit.edu) orP.W.J. (juodawlkis@ II.mit.edu)

\section{A nonlinear optoelectronic filter for electronic signal processing}

\author{
William Loh',2, Siva Yegnanarayanan', Rajeev J. Ram² \& Paul W. Juodawlkis'
}

'Lincoln Laboratory, Massachusetts Institute of Technology, Lexington, Massachusetts, 02420, USA, ${ }^{2}$ Department of Electrical Engineering and Computer Science, Massachusetts Institute of Technology, Cambridge, Massachusetts, 02139, USA.

\begin{abstract}
The conversion of electrical signals into modulated optical waves and back into electrical signals provides the capacity for low-loss radio-frequency (RF) signal transfer over optical fiber. Here, we show that the unique properties of this microwave-photonic link also enable the manipulation of RF signals beyond what is possible in conventional systems. We achieve these capabilities by realizing a novel nonlinear filter, which acts to suppress a stronger RF signal in the presence of a weaker signal independent of their separation in frequency. Using this filter, we demonstrate a relative suppression of $56 \mathrm{~dB}$ for a stronger signal having a 1-GHz center frequency, uncovering the presence of otherwise undetectable weaker signals located as close as $3.5 \mathrm{~Hz}$ away. The capabilities of the optoelectronic filter break the conventional limits of signal detection, opening up new possibilities for radar and communication systems, and for the field of precision frequency metrology.
\end{abstract}

N oise degrades the spectral purity of every oscillator system. Due to the feedback mechanics of oscillators and to the inherent resistance of a saturated oscillator to amplitude fluctuations ${ }^{1}$, phase noise becomes the dominant limitation to performance for nearly all oscillators. The influence of phase noise results in spectral broadening of the oscillation line across a distribution of frequencies. For many systems, the phase noise of a stronger signal can obstruct the detection of weaker signals nearby, burying the weaker signals in noise (Fig. 1a). Ideally, one desires the ability to filter out the stronger signal at the frequency $f_{1}$ leaving behind the signal to be detected at frequency $f_{2}$. However, this operation cannot be realized by conventional all-electronic filters for two reasons. First, the bandwidth of these filters would be insufficiently narrow to separate one signal from another. Second, even with the necessary bandwidth, conventional filters would be unable to distinguish the underlying weaker signal from noise. These limitations have motivated significant effort towards the development of oscillators with extraordinarily low phase noise ${ }^{2-5}$.

Here, we demonstrate a novel nonlinear optoelectronic filter capable of resolving a weaker signal beneath the phase noise of a stronger signal when both received signals are passed through the filter (Fig. 1b). This filter differs from conventional microwave-photonic (MWP) filters ${ }^{6,7}$ as it exploits nonlinearity rather than linear finite impulse response (FIR) or infinite impulse response (IIR) filtering techniques. The nonlinear optoelectronic filter has the configuration of a traditional MWP link ${ }^{8-10}$ consisting of a laser whose output is intensity modulated by a Mach-Zehnder modulator and subsequently detected by a photodetector (Fig. 1c). The RF input voltage applied to the modulator varies the refractive index of the electro-optic material embedded within the modulator. The Mach-Zehnder interferometer converts this linear change in refractive index into a precise sinusoidal variation in the optical intensity. The stored RF information is recovered upon detection of the optical envelope. We show next that under certain ranges of applied RF voltage, this MWP link exhibits properties similar to a filter but with functionality unachievable by ordinary filters. When two signals, one strong and one weak, are passed through the optoelectronic filter, the filter acts to suppress the stronger signal relative to the weaker signal, independent of their separation in frequency (Fig. 1d). The nonlinearity of the system also results in an additional third-order intermodulation product at the filter output.

\section{Results}

Nonlinear optoelectronic filter operation. The filtering behavior of the MWP link is a consequence of an asymmetry in the transmission for the individual signals propagating over the link when their signal amplitudes are different. For a time-varying modulator input voltage comprising the sum of two RF signals $v(t)=v_{1} \sin \left(\omega_{1} t\right)+v_{2} \sin \left(\omega_{2} t\right)$, the resulting modulated optical power $P(t)$ can be expressed as 
a

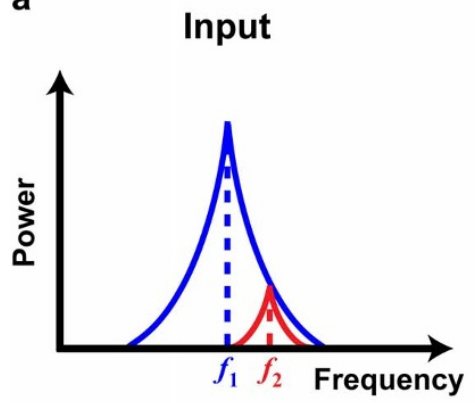

C

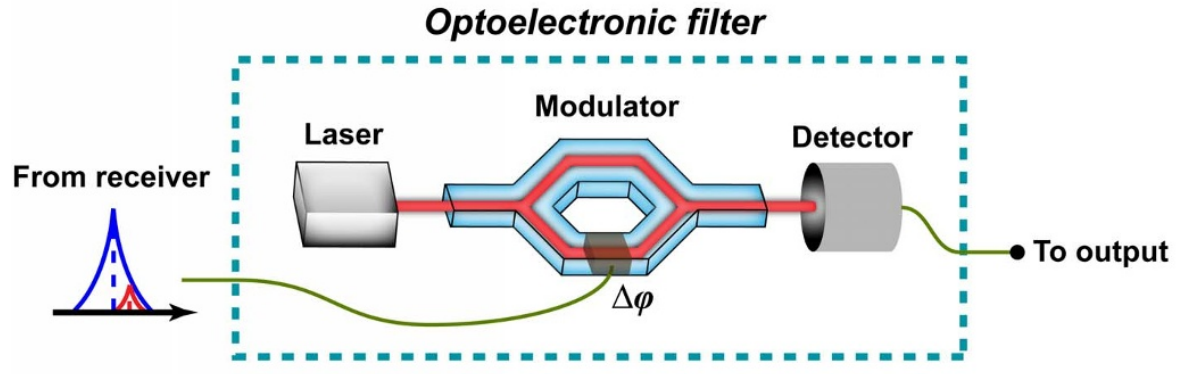

b

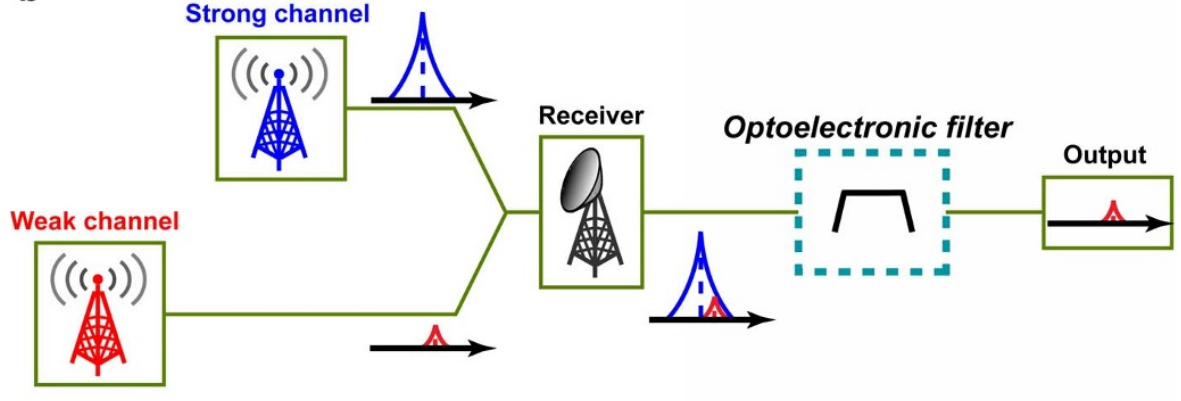

d

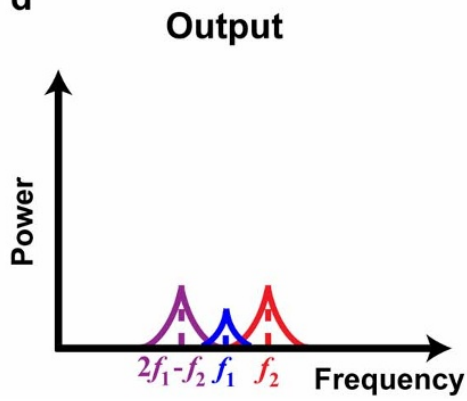

Figure $1 \mid$ The nonlinear optoelectronic filter. (a), Diagram of two input signals consisting of a stronger signal at frequency $f_{1}$ and a weaker signal at frequency $f_{2}$. The weaker signal is buried beneath the phase noise of the stronger signal. (b), The received strong and weak inputs are passed through a nonlinear optoelectronic filter which would ideally act to completely suppress the stronger signal, leaving behind only the weaker signal. (c), The configuration of the nonlinear optoelectronic filter consists of a laser whose optical output is intensity modulated by the received inputs and is subsequently detected by a photodetector. (d), After passing both inputs through the optoelectronic filter, the stronger signal is selectively suppressed, uncovering the presence of the weaker signal. Due to the filter nonlinearity, a third-order $\left(2 f_{1}-f_{2}\right)$ intermodulation product is generated within the frequency band of the inputs.

$$
P(t)=\frac{P_{0}}{2}\left[1+\sin \left(\frac{\pi}{V_{\pi}} v(t)\right)\right]
$$

Here, $v_{1}\left(v_{2}\right)$ and $\omega_{1}\left(\omega_{2}\right)$ are the amplitude and frequency of the first (second) RF signal, $P_{0}$ is the optical power of the laser, and $V_{\pi}$ is the voltage required to transition from constructive to destructive interference in the Mach-Zehnder interferometer. Due to the high linearity exhibited by the electro-optic effect, the variation in optical power with input voltage is nearly a perfect sinusoid. After detection of the modulated power, the generated photocurrent supplied into a load resistor $R$ yields an output voltage

$$
V(t)=P(t) \Re R
$$

where the responsivity $\Re$ is the ratio of the generated photodetector current to the incident optical power. Equation (2) can be expanded into a series of Bessel functions ${ }^{11-13}$ from which the optoelectronic filter transmission can be evaluated for each of the individual inputs (Supplementary Section A). The resulting transmission for each input exhibits saturation depending on the amplitudes of both $v_{1}$ and $v_{2}$, and remarkably this behavior is asymmetric if $v_{1} \neq v_{2}$.

Assuming the case of a strong signal $\left(v_{1}\right)$ and a weak signal $\left(v_{2}\right)$, the magnitude of the voltage transmission for each input can be plotted as a function of $v_{1}$ to determine the saturation induced by the stronger input (Fig. 2a). In Fig. 2a, the transmission characteristics are normalized to unity and $v_{1}$ is normalized to $V_{\pi}$ so that the plotted characteristics remain general across all operating conditions. The voltage transmissions are initially equal for both inputs at low $v_{1}$ but undergo different behaviors as the optoelectronic filter saturates. The transmission behaviors were also verified through measurement, as shown. It is interesting that at the point where $\pi v_{1} / V_{\pi}=3.83$, the theoretical transmission is identically zero for the stronger signal but reaches a local maximum for the weaker signal. The functionality of the optoelectronic filter depends critically on operating at this point. A finer scan around the point of zero transmission is shown in the inset of Fig. 2a where the measurements again verify the nulling of the stronger signal. Although the optoelectronic filter behavior was specifically found assuming low levels of $v_{2}$, it is important to note that the nulling of the transmission for input signal 1 occurs regardless of the value of $v_{2}$ (Supplementary Section A).

Operation at the point of zero transmission allows for unique capabilities in the control of RF signals. For example, if the two inputs of Fig. 1a are sent through the optoelectronic filter with the stronger signal operated at $\pi v_{1} / V_{\pi}=3.83$, the stronger signal can be selectively suppressed relative to the weaker signal independent of their frequency separation. Moreover, this suppression also applies to the phase noise of the stronger signal since the filter transmission remains constant over the range of the signal's frequency excursions. On the other hand, the amplitude fluctuations cannot be completely nulled since the amplitude of the stronger input would vary around the point where $\pi v_{1} / V_{\pi}=3.83$. Fortunately, the phase noise is many orders of magnitude larger than the amplitude noise in almost every oscillator $^{1}$. The optoelectronic filter response can be made to be near instantaneous (limited only by the speed of the modulator and detector) since its operation does not depend on a resonance effect, in contrast to conventional filters. However, the nonlinearities of the optoelectronic filter generate a third-order spurious tone at the frequency $2 f_{1}-f_{2}$ (Fig. 1d). In addition, there will also be spurious signals generated at harmonics of $f_{1}$ that can be readily removed through conventional RF filtering.

Filtering of a stronger signal and its phase noise. The properties of the optoelectronic filter were experimentally verified through measurements of its output electrical spectrum over a span of $100 \mathrm{~Hz}$ (Fig. 2b). The stronger signal was centered at $1 \mathrm{GHz}$, while the weaker signal is located at a frequency larger by $3.5 \mathrm{~Hz}$. The two inputs were operated independently from one another. With both inputs on, the spectrum exhibits the appearance of a single input since the weaker signal is $81.5 \mathrm{~dB}$ below the peak of the stronger 

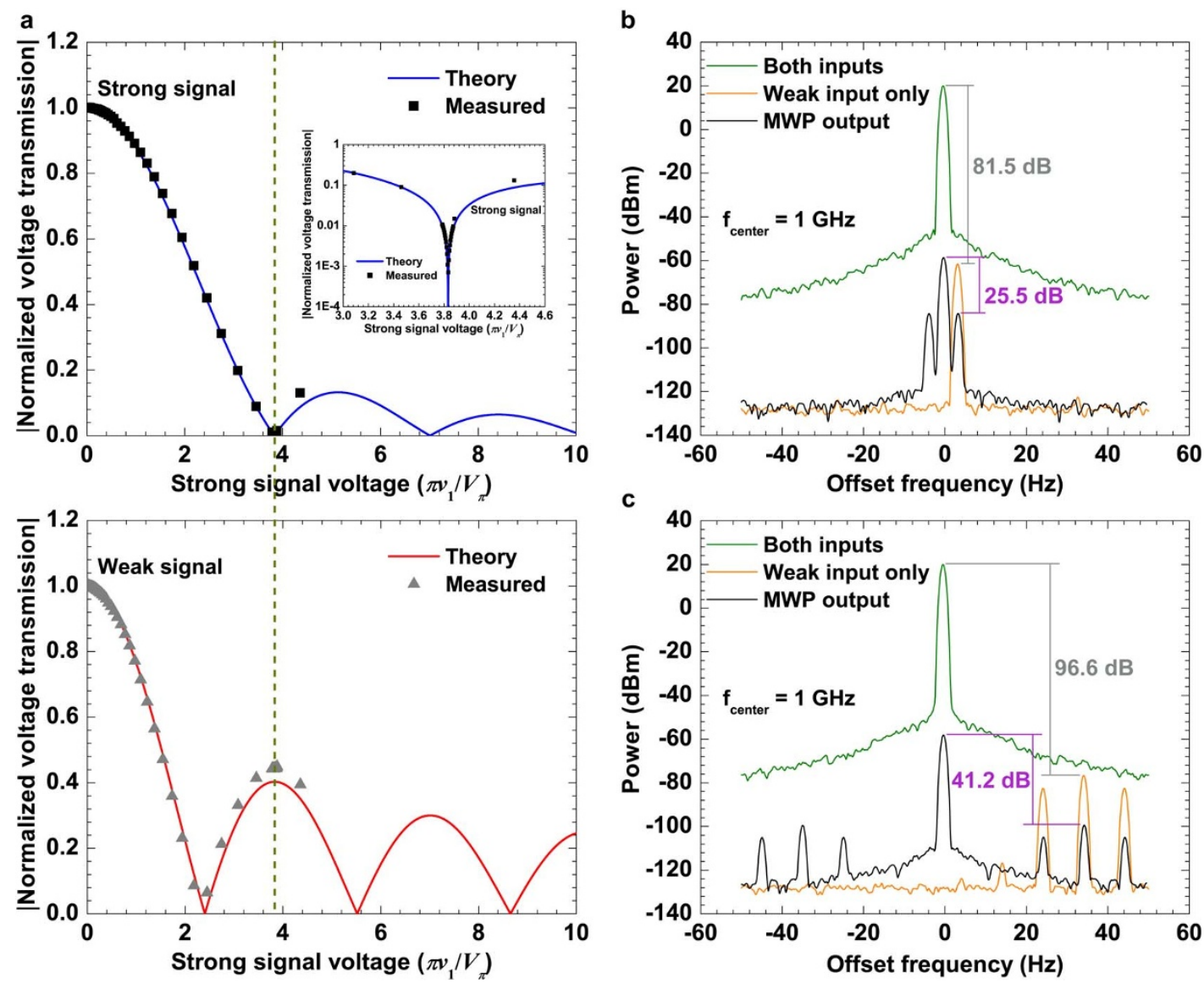

Figure $2 \mid$ Nonlinear filtering of the stronger signal. (a), Measured and theoretical magnitudes of the normalized voltage transmission for a strong and weak signal through the optoelectronic filter. At $\pi v_{1} / V_{\pi}=3.83$, the transmission of the strong signal becomes zero, while the transmission of the weak signal reaches a local maximum. The inset shows a close-up view of the voltage transmission for the stronger signal near the null point. (b), Measured spectrum over a $100 \mathrm{~Hz}$ span at the input and output of the optoelectronic filter. With both inputs on, the spectrum takes the appearance of a single input as the phase noise of the stronger signal masks the presence of the weaker signal. After passing through the filter, the weaker signal becomes clearly visible at a $3.5-\mathrm{Hz}$ frequency offset from the stronger signal. (c), Measured spectra when the weaker signal is amplitude modulated at $10 \mathrm{~Hz}$. The weaker signal and its modulated information are again uncovered after passing through the filter.

signal and $>10 \mathrm{~dB}$ below its phase noise. If the stronger signal is turned off, the weaker signal becomes clearly visible. Conventional filters cannot be used here as they lack both the narrow bandwidth and the ability to reject only the stronger input. However, upon passing both inputs through the nonlinear optoelectronic filter, the stronger signal is selectively suppressed by an additional $56 \mathrm{~dB}$ thus uncovering the presence of the weaker signal. The third-order intermodulation product is also visible on the other side of the spectrum. The total RF loss of the weaker signal was $22.7 \mathrm{~dB}$ here corresponding to operation at a photocurrent of $\sim 4 \mathrm{~mA}$. Since the optoelectronic filter has the dual functionality of a nonlinear filter and an electronic amplifier ${ }^{8}$, net gain can be achieved passing through the filter given a large enough optical power.

Figure $2 \mathrm{c}$ shows a similar measurement but with the weaker signal located $35 \mathrm{~Hz}$ away from the stronger signal and with a RF power that is $96.6 \mathrm{~dB}$ lower. The weaker signal is also amplitude modulated at a frequency of $10 \mathrm{~Hz}$. The spectrum of both inputs again yields the appearance of the stronger input alone since the presence of the weaker signal is masked by phase noise. However, after passing both inputs through the optoelectronic filter, the stronger signal receives an additional $55.4 \mathrm{~dB}$ suppression revealing the underlying weaker signal and its modulation sidebands.

Filtering of a stronger signal under frequency modulation. The above discussion has highlighted the unique capabilities of this optoelectronic filter, resulting in the selective suppression of a stronger input and its corresponding phase noise. The phase-noise suppression is a result of the fact that relatively small fluctuations in frequency do not change the voltage amplitude from operating at $\pi v_{1} / V_{\pi}=3.83$. We show next that these properties also apply to the case of deterministic phase or frequency modulation (Supplementary Section C). For two phase- or frequency-modulated inputs, one strong and one weak, applied to the optoelectronic filter, the operation of the filter acts to suppress the stronger signal and its modulation sidebands (Fig. 3a).

Figure $3 \mathrm{~b}$ shows the measured spectrum of two frequency-modulated inputs sent through the optoelectronic filter. The spectrum spans a width of $20 \mathrm{kHz}$ and is centered on the stronger input located at a frequency of $1 \mathrm{GHz}\left(f_{1}\right)$. The power of the weaker input is $66.4 \mathrm{~dB}$ lower and its frequency is offset from that of the stronger input by $6 \mathrm{kHz}\left(f_{2}\right)$. The weaker signal is frequency modulated at a rate of $1 \mathrm{kHz}$, generating spurs at $7 \mathrm{kHz}$ offset $\left(+f_{\mathrm{m}, 2}\right)$ and $5 \mathrm{kHz}$ offset $\left(-f_{\mathrm{m}, 2}\right)$. On the other hand, the stronger signal is frequency modulated at a rate of $5.6 \mathrm{kHz}$ placing a large modulation sideband $\left(+f_{\mathrm{m}, 1}\right)$ near the middle of the weaker signal. Upon sending both inputs through the optoelectronic filter, the stronger signal and its modulation sidebands decrease uncovering the weaker signal and its modulated information. In addition, the nonlinearities of the optoelectronic filter result in the generation of various spurious tones on the other side of the spectrum.

A similar measurement is provided in Fig. 3c, showing again the spectrum of two frequency-modulated inputs with the weaker signal 


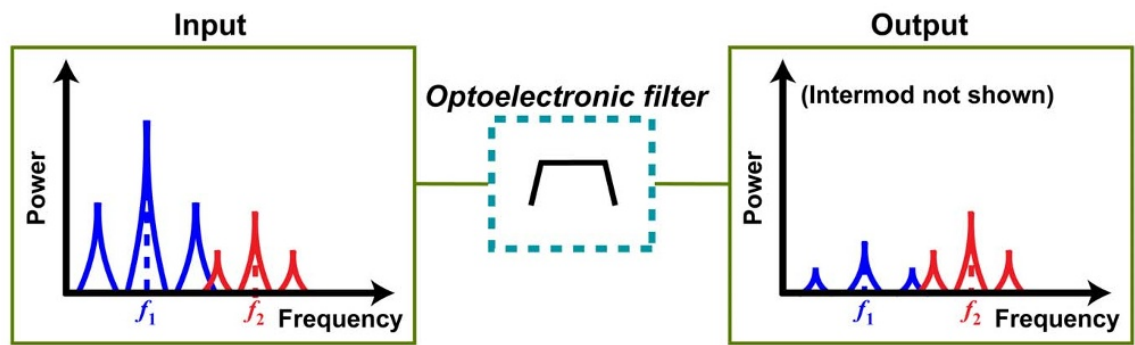

b

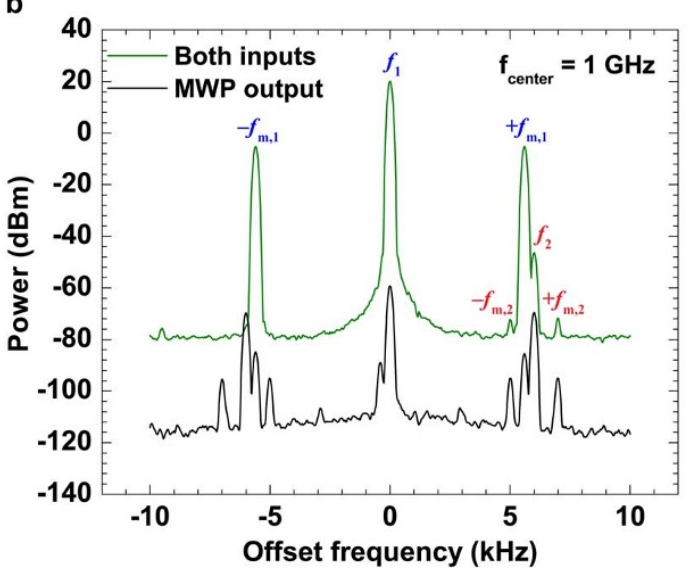

C

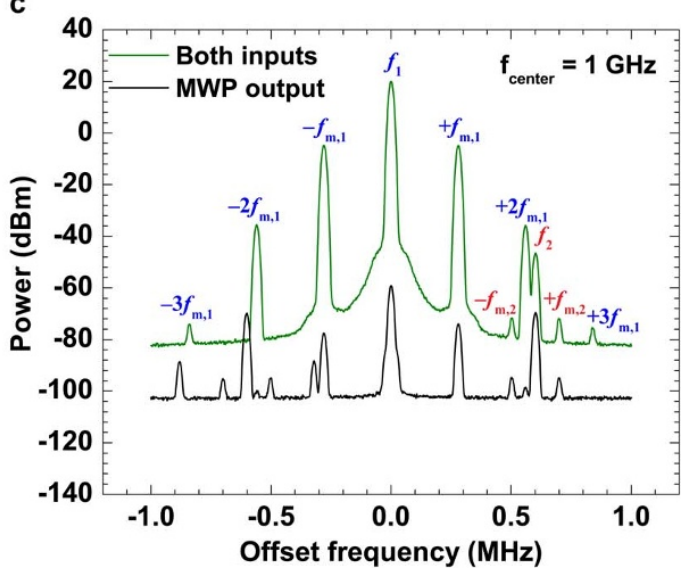

Figure 3 Nonlinear filtering in the presence of frequency modulation. (a), Diagram of two inputs, one strong and one weak, that are frequency modulated and sent through a nonlinear optoelectronic filter. The filter acts to suppress the stronger input and its modulation sidebands relative to the weaker input. (b), Measured spectrum $(20 \mathrm{kHz}$ span) at the input and output of the optoelectronic filter with the input signals and their modulation sidebands indicated. The large modulation sideband of the stronger signal $\left(+f_{\mathrm{m}, 1}\right)$ initially obstructs the detection of the weaker signal but becomes greatly suppressed after passing through the filter. (c), Measured spectra over a $2-\mathrm{MHz}$ span with the second sideband of the stronger signal $\left(+2 f_{\mathrm{m}, 1}\right)$ initially blocking the detection of the weaker signal. After passing through the optoelectronic filter, this sideband becomes suppressed nearly to the level of the background noise.

$66.5 \mathrm{~dB}$ below the stronger signal. The spectrum is centered on the $1 \mathrm{GHz}$ stronger signal but now spans a width of $2 \mathrm{MHz}$. The weaker signal is offset in frequency by $600 \mathrm{kHz}$ and is frequency modulated at a rate of $100 \mathrm{kHz}$. As before, its upper and lower modulation sidebands are denoted by $+f_{\mathrm{m}, 2}$ and $-f_{\mathrm{m}, 2}$, respectively. The stronger signal is frequency modulated at a rate of $280 \mathrm{kHz}$, generating multiple sidebands $\left( \pm f_{\mathrm{m}, 1}\right.$ through $\left.\pm 3 f_{\mathrm{m}, 1}\right)$ within the $2 \mathrm{MHz}$ span. The $+2 f_{\mathrm{m}, 1}$ sideband is located near the center of the weaker signal obstructing the ability to detect the modulated information. However, after passing both inputs through the optoelectronic filter, only a small remnant of the $+2 f_{\mathrm{m}, 1}$ sideband can still be observed. This suppression applies also to the stronger signal itself and to the rest of its modulation sidebands.

\section{Discussion}

The asymmetric transmission for the individual signals through the nonlinear optoelectronic filter allow for unique capabilities in the control of electrical signal behavior. We demonstrated that by operating at the transmission null, a stronger input and its corresponding phase fluctuations or frequency modulation can be selectively suppressed relative to a weaker input. This suppression is independent of the frequency separation between the stronger and weaker signals and is not limited by the propagation time through a filter resonance. Furthermore, the optoelectronic filter can even provide net gain given sufficient optical power from the laser. These properties are especially useful in radar and precision metrology applications for detecting minute traces of a desired target over a large interferer signal. In communication systems, the ability to selectively suppress a modulated channel would allow for information packing at greater densities and gives rise to the possibility of sending hidden signals detectable only by the optoelectronic filter.
The simplicity of the scheme makes the optoelectronic filter amenable to photonic integration. Automatic gain control can be employed to lock the stronger input to its transmission null. In addition, techniques of sampling and digital signal processing or RF downconversion and analog filtering can be employed to remove unwanted intermodulation products. Note that because the modulator $V_{\pi}$ varies slightly with frequency, the condition $\pi v_{1} / V_{\pi}=3.83$ becomes more difficult to maintain when the modulation sidebands become too far separated from the center. In these cases, the suppression of the sidebands becomes reduced. However, these issues can be mitigated through the use of modulators having wider bandwidth. The additional functionality provided by the nonlinear optoelectronic filter over conventional filters significantly enhances the ability of systems to detect weak signals.

\section{Methods}

Optoelectronic filter components. The components of the optoelectronic filter consisted of a JDS Uniphase CQF935 distributed feedback laser $\left(P_{0}=20 \mathrm{~mW}\right)$, a $14 \mathrm{GHz}$ EOSPACE intensity modulator $\left(V_{\pi}=2.66 \mathrm{~V}\right.$ at $\left.1 \mathrm{GHz}\right)$, and a $4.7 \mathrm{GHz}$ Discovery Semiconductors DSC50 photodiode $(\Re=0.77 \mathrm{~A} / \mathrm{W})$. Polarization paddles were used before the modulator for control of the input polarization, and two optical isolators were employed to prevent unwanted reflections, one after the laser and one before the photodetector.

Measurements of strong-signal suppression. The measurements were performed by modulating the laser power with an RF input consisting of two signals produced from independent RF frequency synthesizers. The RF power and frequency of each signal was controlled by setting each synthesizer with no additional locking required. The photodetected output of the optoelectronic filter was then sent through a DC block and into an Agilent E4440A spectrum analyzer for processing. By measuring the traces at both the input and output of the link, the transmission can be determined for both inputs. The spectrum measurement also captures the presence of nonlinear intermodulation products. A total of three RF sources were used to generate the desired signals transmitted through the optoelectronic filter. For the measurements 
that did not require frequency modulation, an Agilent E8241A signal generator served as the stronger input while a HP 8665B signal generator served as the weaker input. For the measurements employing frequency modulation, the HP 8665B signal generator with its internal modulation capabilities served as the stronger input amplified by a Mini-Circuits ZHL-42W RF amplifier. A HP 8340B signal generator served as the weaker input externally modulated by a Tektronix FG504 function generator.

1. Loh, W., Yegnanarayanan, S., Ram, R. J. \& Juodawlkis, P. W. Unified theory of oscillator phase noise I: white noise. IEEE Trans. Microw. Theory Tech. 61 2371-2381 (2013).

2. Yao, X. S. \& Maleki, L. Optoelectronic microwave oscillator. J. Opt. Soc. Am. B 13 , 1725-1735 (1996)

3. Yao, X. S. \& Maleki, L. Optoelectronic oscillator for photonic systems. IEEE J. Quantum Electron. 32, 1141-1149 (1996).

4. Maleki, L. Sources: the optoelectronic oscillator. Nature Photon. 5, 728-730 (2011).

5. Fortier, T. M. et al. Generation of ultrastable microwaves via optical frequency division. Nature Photon. 5, 425-429 (2011).

6. Capmany, I., Ortega, B. \& Pastor, D. A tutorial on microwave photonic filters. IEEE J. Lightwave Technol. 24, 201-229 (2006).

7. Minasian, R. A. Photonic signal processing of microwave signals. IEEE Trans. Microw. Theory Tech. 54, 832-846 (2006).

8. Cox, C. H. Analog Optical Links: Theory and Practice (Cambridge University Press, 2004)

9. Chang, W. S. RF Photonic Technology in Optical Fiber Links (Cambridge University Press, 2002)

10. Capmany, J. \& Novak, D. Microwave photonics combines two worlds. Nature Photon. 1, 319-330 (2007).

11. Kolner, B. H. \& Dolfi, D. W. Intermodulation distortion and compression in an integrated electrooptic modulator. Appl. Opt. 26, 3676-3680 (1987).
12. Olshansky, R. Optimal design of subcarrier multiplexed lightwave systems employing linearized external modulators. IEEE J. Lightwave Technol. 10, 378-382 (1992)

13. Loh, W., Yegnanarayanan, S., Ram, R. J. \& Juodawlkis, P. W. Super-homogeneous saturation of microwave-photonic gain in optoelectronic oscillator systems. IEEE Photon. J. 4, 1256-1266 (2012).

\section{Acknowledgments}

This work was supported by the Assistant Secretary of Defense for Research and Engineering under Air Force contract \#FA8721-05-C-0002.

\section{Author contributions}

W.L. conceived the idea of this study, developed the theory, and performed the measurements. S.Y., R.J.R. and P.W.J. supervised this study. All authors contributed to the designing of the experiments and to the writing of this manuscript.

\section{Additional information}

Supplementary information accompanies this paper at http://www.nature.com/ scientificreports

Competing financial interests: The authors declare no competing financial interests

How to cite this article: Loh, W., Yegnanarayanan, S., Ram, R.J. \& Juodawlkis, P.W. A nonlinear optoelectronic filter for electronic signal processing. Sci. Rep. 4, 3613; DOI:10.1038/srep03613 (2014)

(c) (i) (2) This work is licensed under a Creative Commons AttributionBY Nc SA NonCommercial-ShareAlike 3.0 Unported license. To view a copy of this license, visit http://creativecommons.org/licenses/by-nc-sa/3.0 